\title{
Theory, Design, and Characterization of Nanoelectromechanical Relays for Stiction-Based Non-Volatile Memory
}

\author{
Dinesh Pamunuwa ${ }^{\circledR}$, Senior Member, IEEE, Elliott Worsey, Jamie D. Reynolds, \\ Derek Seward, Harold M. H. Chong, and Sunil Rana
}

\begin{abstract}
Diverse areas such as the Internet of Things (IoT), aerospace and industrial electronics increasingly require non-volatile memory to work under high-temperature, radiationhard conditions, with zero standby power. Nanoelectromechanical (NEM) relays uniquely have the potential to work at $300{ }^{\circ} \mathrm{C}$ and absorb high levels of radiation, with zero leakage current across the entire operational range. While NEM relays that utilise stiction for non-volatile operation have been demonstrated, it is not clear how to design a relay to reliably achieve given programming and reprogramming voltages, an essential requirement in producing a memory. Here, we develop an analytical, first-principle physics-based model of rotational NEM relays to provide detailed understanding of how the programming and reprogramming voltages vary based on the device dimensions and surface adhesion force. We then carry out an experimental parametric study of relays with a critical dimension of $\approx 80 \mathrm{~nm}$ to characterise the surface adhesion force, and derive guidelines for how a NEM relay should be dimensioned for a given contact surface force, feature size constraints and operating requirements. We carry out a scaling study to show that voltages of $\approx 1 \mathrm{~V}$ and a footprint under $\approx 2 \mu \mathrm{m}^{2}$ can be achieved with a critical dimension of $\approx 10 \mathrm{~nm}$, with this device architecture.

[2021-0138]
\end{abstract}

Index Terms-Nanoelectromechanical (NEM) systems, nanofabrication, nonvolatile memory, microelectromechanical devices, high-temperature.

Manuscript received June 28, 2021; revised December 3, 2021; accepted December 20, 2021. The work of Dinesh Pamunuwa was supported in part by the Royal Academy of Engineering Senior Research Fellowship under Grant RCSRF1920-9-53. This work was supported in part by the European Union (EU) H2020 Research and Innovation Programme under Agreement 871740 (ZeroAMP), in part by the University of Bristol Cleanroom Facility through the U.K. Engineering and Physical Sciences Research Council (EPSRC) Grant EP/N015126/1 (QuPIC), and in part by the Nanofabrication Centre at the University of Southampton through the EPSRC Platform Grant EP/N013247/1. Subject Editor A. Seshia. (Corresponding author: Dinesh Pamunuwa.)

Dinesh Pamunuwa, Elliott Worsey, and Sunil Rana are with the Department of Electrical and Electronic Engineering, University of Bristol, Bristol BS8 1UB, U.K. (e-mail: dinesh.pamunuwa@bristol.ac.uk).

Jamie D. Reynolds is with the School of Electronics and Computer Science, University of Southampton, Southampton SO17 1BJ, U.K.

Derek Seward is with the Department of Engineering, Lancaster University, Lancaster LA1 4YW, U.K.

Harold M. H. Chong is with the School of Electronics and Computer Science, University of Southampton, Southampton SO17 1BJ, U.K., and also with the Japan Advanced Institute of Science and Technology, Ishikawa 923-1292, Japan.

Digital Object Identifier 10.1109/JMEMS.2021.3138022

\section{INTRODUCTION}

$\mathbf{E}$ LECTRONIC memory, especially non-volatile memory that retains the switched state when power is switched off, is a key part of any electronic system. Solid-state nonvolatile memory is temperature-limited and particularly vulnerable to radiation upsets. Nanoelectromechanical (NEM) relays, by contrast, have zero sleep current, a steep subthreshold slope [1]-[6], as well as the capability to operate at elevated temperatures [7] and radiation levels [8] where transistors either work suboptimally or not at all. Non-volatile operation of NEM relays has been demonstrated using a variety of designs and schemes, including charge storage in a floating gate to alter the pull-in voltage [9], [10], and stiction between contacting surfaces [11]-[13]. We recently demonstrated a stiction-based bistable NEM relay with a semicircular beam that eliminates electromechanical pull-in instability [14], allowing precise electrostatic control of the beam when switching between two stable states (please see Table 1 in [14] for a comparison between electrostatic nonvolatile relays). As a result, the device has great potential to serve as a non-volatile memory cell. In order to produce a working memory, however, more understanding and validation of the stiction-based non-volatile functionality as well as design guidelines are essential.

Here, we develop from first principles, an analytical physicsbased model of the device governing programming and reprogramming, and carry out an experimental parametric study to that end. We investigate the performance of different contact designs and characterise the surface adhesion force from the pull-out voltages and investigate the threshold between nonvolatile and volatile functionality. We also provide detailed analyses on device operation, discuss its design and carry out a scaling study. The methodology and analysis also has wider implications than the design of one type of relay, as the work establishes fundamental knowledge of the operation of all stiction-based non-volatile switches, an important and widely researched area.

\section{NEM SWITCH FUNCTIONALITY AND THEORY OF OPERATION}

The rotational NEM relay considered here [14] consists of a semicircular beam anchored at or near its geometric 
(a)

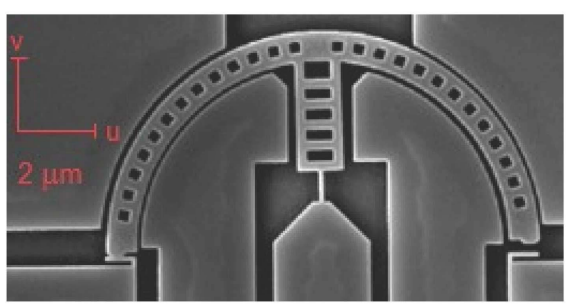

(c)

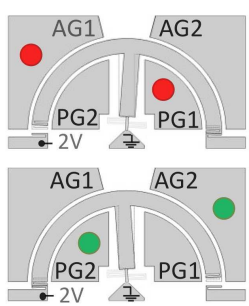

(d)

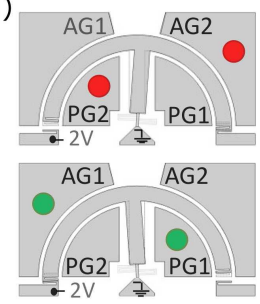

(b)

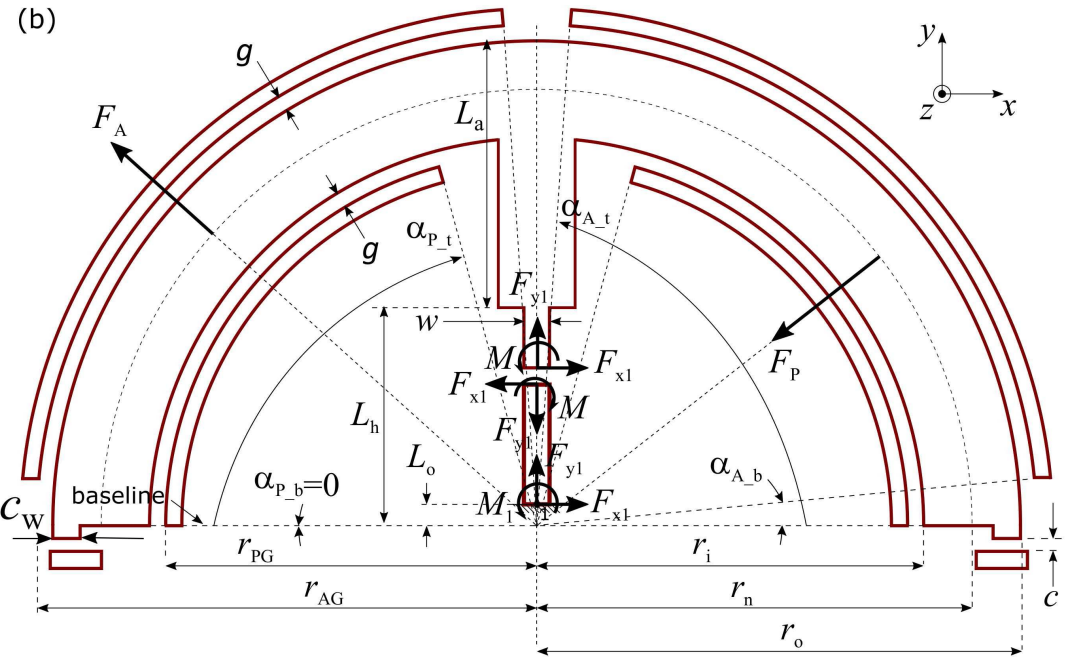

Fig. 1. Rotational moment-driven relay with in-plane, quad-gate actuation. (a) Fabricated nanorelay with $120 \mathrm{~nm}$ actuation airgap $(\mathrm{g})$ and $80 \mathrm{~nm}$ hinge width $(w)$ comprising principal $(\mathrm{PG})$ and auxiliary $(\mathrm{AG})$ gates that are actuated in pairs. (b) Detailed geometry of relay with positive hinge offset, and electrostatic forces $F_{P}$ and $F_{A}$ resulting from actuation pattern at top of (c), to rotate relay clockwise. (c) and (d) Actuation patterns for relays with positive and negative hinge offsets respectively, to rotate relay clockwise (top) and anticlockwise (bottom) where the coloured circles represent application of the actuation voltage.

center, and four arcuate gates where the beam and gate arcs are concentric (see Fig. 1(a) for an example of a fabricated device). The inner gates are termed principal gates (PG1 and PG2), and the outer gates auxiliary gates (AG1 and AG2), and the full device geometry is defined in Fig. 1(b), with the parameters defined in Table I. In general, the hinge anchor point can be above (positive hinge offset) or below (negative hinge offset) the baseline diameter of the beam semicircle. The beam rotates when the actuation voltage is applied to one principal and auxiliary gate pair. The actuation pattern to rotate the relay in a given direction depends on the hinge offset $L_{0}$. If $L_{\mathrm{o}}$ is greater than a critical hinge offset $L_{\mathrm{c}}$, driving gate pair 1 (i.e. PG1 and AG1) results in clockwise rotation of the beam, while driving gate pair 2 results in anticlockwise rotation (shown in 1(c)). If, however, $L_{\mathrm{o}}<L_{\mathrm{c}}$, the actuation patterns are reversed, i.e. clockwise rotation is achieved by driving gate pair 2, while anticlockwise rotation is achieved by driving gate pair 1 (Fig. 1(d)). The advantage conferred by this architecture is that a near constant airgap is maintained as the beam rotates, and thus electromechanical pull-in instability is avoided, as discussed in our previous work [14]. In the following sections we develop from first principles an entirely new model governing both programming and reprogramming using a simpler, yet more accurate and physically intuitive approach than the original model (see [14], supplementary material).

\section{A. Actuation Model}

When an actuation voltage $V$ is simultaneously applied to a principal and auxiliary gate pair, the electrostatic forces are determined by the capacitance between the beam and actuated gate pair:

$$
F=\frac{\alpha \epsilon_{0} t V^{2}}{2 b[\ln (b / a)]^{2}}
$$

where $\epsilon_{0}$ is the free space permittivity and $t$ is the thickness of the relay beam and gates. For the inner capacitance and force
$F_{\mathrm{P}}, b$ is the inner radius of the circular beam $r_{\mathrm{i}}, a$ the radius of the principal gates $r_{\mathrm{PG}}$ and $\alpha=\alpha_{\mathrm{P}}=\alpha_{\mathrm{P}_{-} \mathrm{t}}-\alpha_{\mathrm{P} \_\mathrm{b}}$, defined as the span angle of the principal gate (Fig. 1(b)). For the outer capacitance and force $F_{\mathrm{A}}, b$ is the radius of the auxiliary gate $r_{\mathrm{AG}}, a$ is the outer radius of the beam, $r_{\mathrm{O}}$ and the span angle is $\alpha=\alpha_{\mathrm{A}}=\alpha_{\mathrm{A} \_\mathrm{t}}-\alpha_{\mathrm{A} \_\mathrm{b}}$. The curved beam and straight central arm are both much wider than the hinge, and thus the relay rotation is modelled as entirely dependent on the deflection of the hinge. The hinge anchor point (labelled ' 1 ' in Fig. 1(a)) is the $(x, y)$ coordinate origin, and may be above or below the horizontal arc diameter (baseline) depending on the hinge offset. For horizontal and vertical equilibrium of the relay,

$$
\begin{aligned}
& F_{\mathrm{x} 1}=F_{\mathrm{A}} \times 1 / 2\left(\cos \alpha_{\mathrm{A}}\right)+F_{\mathrm{P}} \times 1 / 2\left(\cos \alpha_{\mathrm{P}}\right) \\
& F_{\mathrm{y} 1}=F_{\mathrm{P}} \times 1 / 2\left(\sin \alpha_{\mathrm{P}}\right)-F_{\mathrm{A}} \times 1 / 2\left(\sin \alpha_{\mathrm{A}}\right) .
\end{aligned}
$$

Taking moments about point 1 for the relay,

$$
M_{1}=F_{\mathrm{X} 1} L_{\mathrm{o}} .
$$

Using eq. (4), the internal bending moment $M$ at distance $y$ from the anchor point is

$$
M=F_{\mathrm{x} 1}\left(L_{\mathrm{o}}+y\right) \text {. }
$$

Thus, under the assumption of small deflections,

$$
E I \frac{d^{2} x}{d y^{2}}=F_{\mathrm{x} 1}\left(L_{\mathrm{o}}+y\right)
$$

where $I=\frac{w^{3}}{12 t}$ is the second moment of area of the rectangular hinge cross section for bending along the $y$ axis and $E$ is Young's modulus for Si. Hence, the deflection $x$ at a distance $y$ along the hinge (given that $x=0, d x / d y=0$ at $y=0$ ) is:

$$
x=\frac{F_{\mathrm{x} 1}}{E I}\left(L_{\mathrm{o}} \frac{y^{2}}{2}+\frac{y^{3}}{6}\right) .
$$

From geometrical considerations, the angle $\theta_{\mathrm{a}}$ inscribed by the arc of the beam at its geometric centre is equal to the 
TABLE I

Nominal Geometrical PARAmeters for the RElay

\begin{tabular}{l|l|l}
\hline Thickness (SOI wafer device layer & $t$ & $300 \mathrm{~nm}$ \\
thickness) & $g$ & $120 \mathrm{~nm}$ \\
Actuation airgap & $c$ & $100 \mathrm{~nm}$ \\
Contact airgap & $\alpha_{\mathrm{P}_{-} \mathrm{b}}$ & $0^{\circ}$ \\
Base angle of Principal Gate & $\alpha_{\mathrm{P} \_\mathrm{t}}$ & $75^{\circ}$ \\
Top angle of Principal Gate & $\alpha_{\mathrm{P}}$ & $\alpha_{\mathrm{P}_{\mathrm{t}}}-\alpha_{\mathrm{P} \_\mathrm{b}}$ \\
Span angle of Principal Gate & $\alpha_{\mathrm{A} \_\mathrm{b}}$ & $5^{\circ}$ \\
Base angle of Auxiliary Gate & $\alpha_{\mathrm{A} \_\mathrm{t}}$ & $65^{\circ}$ \\
Top angle of Auxiliary Gate & $\alpha_{\mathrm{A}}$ & $\alpha_{\mathrm{A} \_\mathrm{t}}-\alpha_{\mathrm{A} \_\mathrm{b}}$ \\
Span angle of Auxiliary Gate & $r_{\mathrm{i}}$ & $5.0 \mu \mathrm{m}$ \\
Inner radius & $r_{\mathrm{o}}$ & $5.8 \mu \mathrm{m}$ \\
Outer radius & $r_{\mathrm{PG}}$ & $5.92 \mu \mathrm{m}$ \\
Radius of Principal Gate & $r_{\mathrm{AG}}$ & $4.88 \mu \mathrm{m}$ \\
Radius of Auxiliary Gate & $W_{\mathrm{s}}$ & $1.25 \mu \mathrm{m}$ \\
Width of straight central beam & $W_{\mathrm{c}}$ & $0.8 \mu \mathrm{m}$ \\
Width of semicircular arms & $L_{\mathrm{h}}$ & $0.84 \mu \mathrm{m}$ \\
Hinge length & $L_{\mathrm{o}}$ & variable $(-1$ to $1.2 \mu \mathrm{m})$ \\
Hinge offset & $L_{\mathrm{c}}$ & $-L_{\mathrm{h}} / 2$ \\
Hinge offset at which no rotation & \\
occurs & $L_{\mathrm{a}}$ & variable \\
Straight arm length & & $5.16 \mu \mathrm{m})$ \\
& $w$ & variable $(75 \mathrm{to} 100 \mathrm{~nm})$ \\
Width of hinge & $c_{\mathrm{W}}$ & 50 and $100 \mathrm{~nm}$ \\
Contact width & & 'T' or 'S' \\
Contact type &
\end{tabular}

slope of the hinge tip with the vertical (Fig. 2(a)). For an infinitesimally small angle $d \theta$,

$$
\int d \theta=\frac{1}{E I} \int M d y=\frac{F_{\mathrm{x} 1}}{E I} \int\left(L_{\mathrm{o}}+y\right) d y
$$

Integrating eq. (8) and using the condition that $\theta=0$ at $y=0$ gives the expression for $\theta$ as a function of $y$ :

$$
\theta=\frac{F_{\mathrm{x} 1}}{E I}\left(L_{\mathrm{o}} y+\frac{y^{2}}{2}\right) .
$$

From eq. (7), the point along the hinge at which the deflection is zero is $y=-3 L_{0}$, while eq. (9) gives the points along the hinge at which the slope is zero and a minimum respectively as $y=-2 L_{\mathrm{o}}$ and $y=-L_{\mathrm{o}}$. Thus, for a constant hinge length $L_{\mathrm{h}}$, the hinge offset $L_{\mathrm{o}}$ fundamentally affects how the relay rotates, and in turn the rotation voltage, as described in the following section.

\section{B. Beam Rotation}

The variation of slope $\theta$ with $y$ for a given force $F_{x 1}$, eq. (9), is plotted in Fig. 2(b) for zero and positive hinge offsets (left) and negative hinge offsets (right). For a constant hinge length $L_{\mathrm{h}}$, the vertical arm length $L_{\mathrm{a}}$ (see Fig. 2(a)) changes to accommodate different hinge offsets. As $x=0$, $y=0$, is always the anchor point (labelled as 1), the physical constraint on $y$ is that $0 \leq y \leq L_{\mathrm{h}}$. Thus, based on the hinge offset and resulting allowable values for $y$, five distinct regimes of operation, R1 through R5, can be identified. For each regime, $y$ can take any value from the origin to the righthand boundary of the relevant shaded area, and the value of the curve on the right-hand boundary gives the slope at the top of the hinge for that regime. For example, for zero and positive hinge offsets (regime R1), $y$ can take any value from 0 to $L_{\mathrm{h}}$, and the slope at the top of the hinge is given by the righthand boundary of the region shaded green (Fig. 2(b), left).
The higher the offset, the more negative the $y$ value at which the curve's minimum occurs. For zero offset, the curve is symmetrical about the $y$ axis of the graph.

Similar to positive hinge offsets, the right-hand boundary of the shaded regions in Fig. 2(b), right, correspond to the top of the hinge for each of the different regimes resulting from negative hinge offsets. Thus, for example, for $\left|L_{\mathrm{o}}\right|<L_{\mathrm{h}} / 3$ (regime R2), $y$ can take any value from 0 to the right-hand boundary of the R2 shaded region. The area of the box represents the space allowed by the inequality; the closer the hinge offset magnitude $\left|L_{\mathrm{o}}\right|$ is to $L_{\mathrm{h}} / 3$, the narrower the R2 region. Similarly, for $L_{\mathrm{h}} / 3<\left|L_{\mathrm{o}}\right|<L_{\mathrm{h}} / 2$ (regime R3), $y$ can take any value from 0 to the right-hand boundary of the shaded R3 region. As a final example, if $\left|L_{\mathrm{o}}\right|>L_{\mathrm{h}}$ (regime R5), the largest possible $y$ value is $y=-L_{0}$ (in order to be less than the hinge length), and the possible values for $y$ are from 0 to the right-hand boundary of the R5 box. The labelling is such that the order of the regimes increases as the hinge offset becomes more negative, and each of the five regimes are discussed below. The relay geometry used in this analysis is the same as in our fabrication experiments described later, and is specified in Table I (symbols are defined in Fig. 1(b)). In particular, as the regimes are predicated on the offset as a fraction of $L_{\mathrm{h}}$, it is worth noting that hinge length $L_{\mathrm{h}}=0.84 \mu \mathrm{m}$.

1) R1-Zero/Positive Hinge Offsets: $L_{\mathrm{o}} \geq 0$ : For $L_{\mathrm{o}} \geq 0$, the hinge experiences a positive bending moment along its entire length (see eq. (5)). Thus, the hinge bends in the same direction with a gradually increasing slope as sketched in Fig 2(a), R1. The deflection curve corresponding to $L_{\mathrm{o}}=0$ (green) and increasing positive offsets (red, blue and black) are shown in Fig. 2(c), left. The arc of rotation described by the beam (see Fig. 2(a) R1) is $S_{1}+S_{2}$ where $S_{1}=0.5\left(L_{\mathrm{h}}+L_{\mathrm{a}}\right) \theta_{\mathrm{a}}$ and $S_{2}=0.5 L_{\mathrm{a}} \theta_{\mathrm{a}}$ for $\theta_{\mathrm{a}}=\frac{F_{\mathrm{x} 1}}{E I}\left(L_{\mathrm{o}} L_{\mathrm{h}}+\frac{L_{\mathrm{h}}^{2}}{2}\right)$ (using eq. (9) which gives the slope at the end of the hinge with the vertical).

2) R2-Negative Hinge Offsets: $L_{\mathrm{o}}<0, \quad\left|L_{\mathrm{o}}\right|<L_{\mathrm{h}} / 3$ : In this regime, the section of the hinge below the baseline experiences a negative bending moment while the rest of the hinge sees a positive bending moment. Thus, the hinge starts off bending to the left, and then bends to the right, and the final slope is positive as sketched in Fig. 2(a), R2. The deflection is zero at $y=-3 L_{0}$, using eq. (7). The deflection plot for $L_{\mathrm{o}}=$ $-0.28 \mu \mathrm{m}$ in Fig. 2(c), right, shows how the slope changes from negative to positive. By considering the sum of the three angles at point $\mathrm{P}$ in Fig. 2(a), R2, $\alpha=\frac{\theta_{\mathrm{a}}-\theta_{\mathrm{b}}}{2}$. The deflection angles are $\theta_{\mathrm{a}}=\frac{F_{\mathrm{X} 1}}{E I}\left(L_{\mathrm{o}} L_{\mathrm{h}}+\frac{L_{\mathrm{h}}^{2}}{2}\right)$ and $\theta_{\mathrm{b}}=\frac{3 F_{\mathrm{X} 1}}{2 E I} L_{\mathrm{o}}{ }^{2}$, using eq. (9). Now, the arc described by the beam is $S_{1}+S_{2}$ where $S_{1}=\left(L_{\mathrm{h}}-3\left|L_{\mathrm{o}}\right|+L_{\mathrm{a}}\right)\left(\theta_{\mathrm{a}}-\alpha\right)$ and $S_{2}=L_{\mathrm{a}} \alpha$.

3) R3-Negative Hinge Offsets: $L_{\mathrm{o}}<0, L_{\mathrm{h}} / 3<\left|L_{\mathrm{o}}\right|<$ $L_{\mathrm{h}} / 2$ : As before, the hinge experiences a negative bending moment for the section below the base line, and a positive bending moment for the rest. Thus, also as before, the hinge starts off bending to the left, and then bends to the right, with the final slope being positive (sketched in Fig. 2(a), R3). This again results in clockwise rotation. The difference is that the net deflection is now either zero (deflection curve 

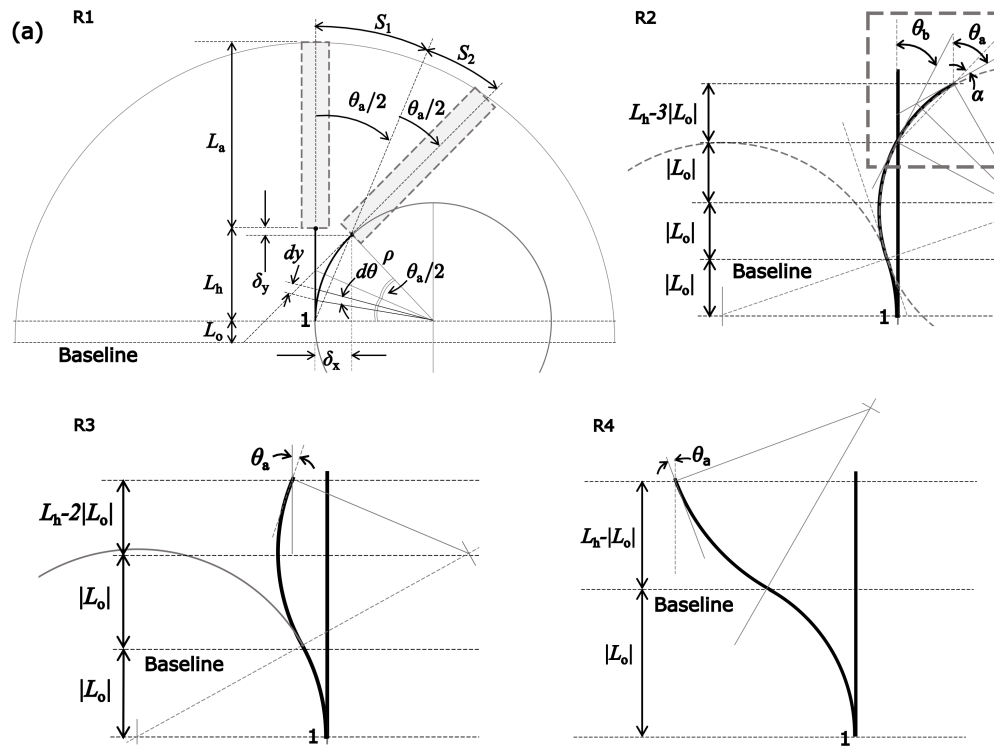

(b)

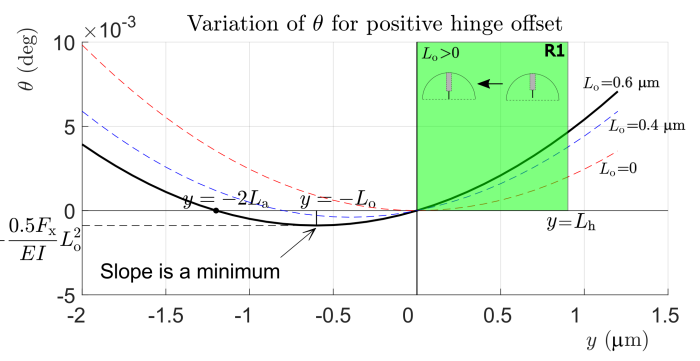

(c)

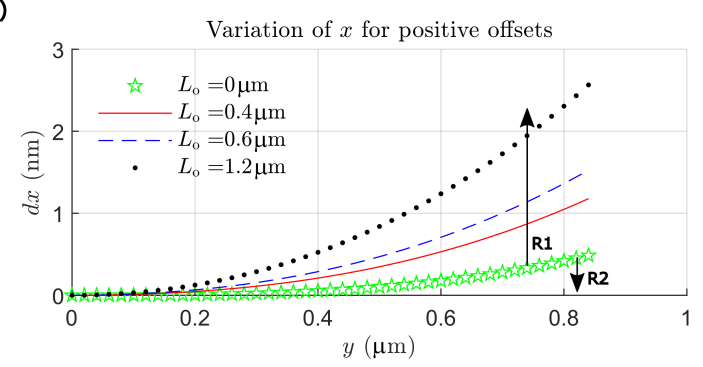

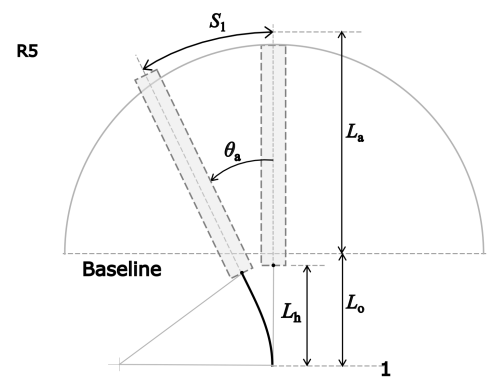
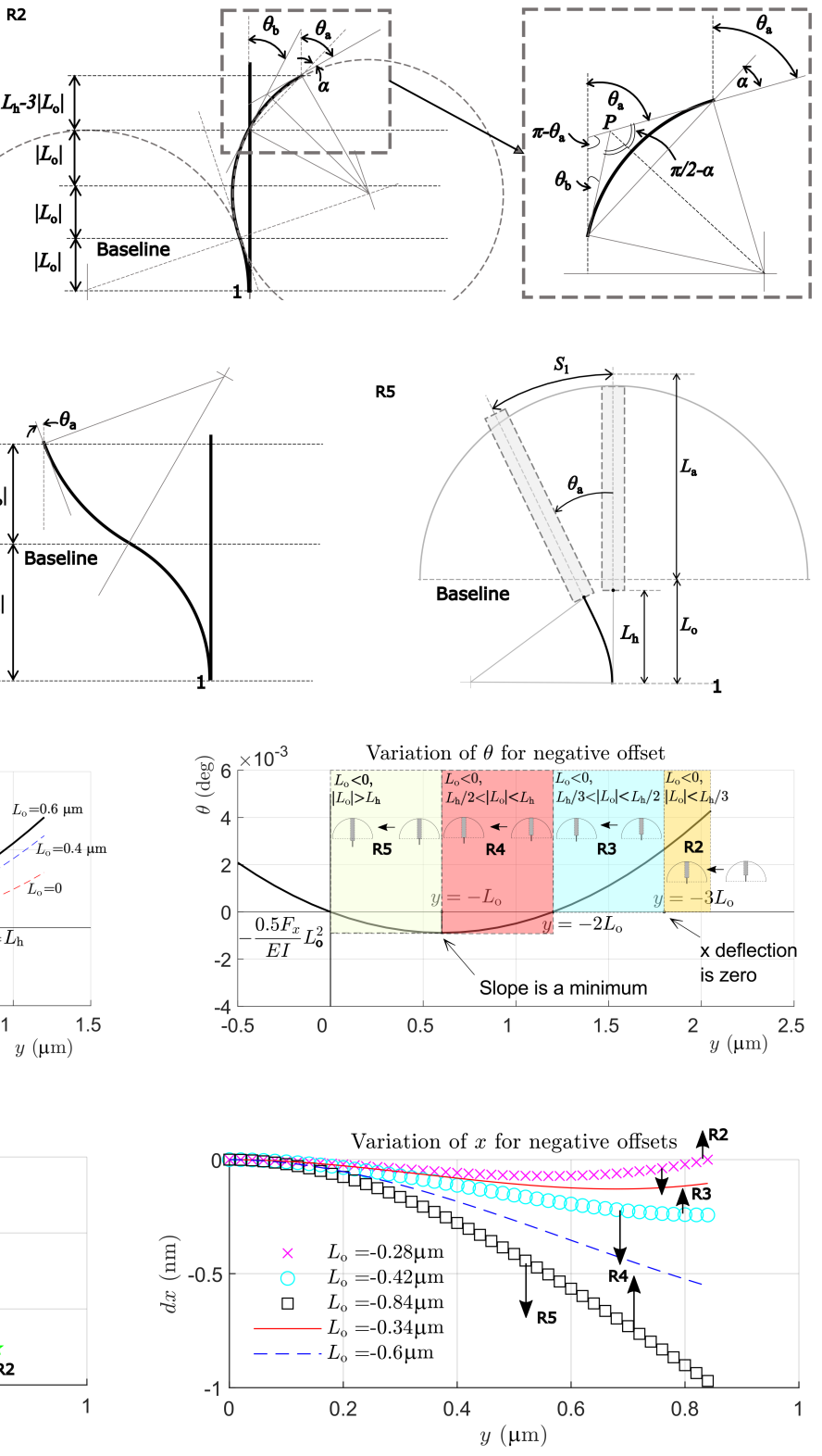

Fig. 2. Operating regimes of relay for different hinge offsets. Regimes are defined as R1: $L_{\mathrm{O}} \geq 0, \mathrm{R} 2: 0<-L_{\mathrm{O}}<\frac{L_{\mathrm{h}}}{3}, \mathrm{R} 3$ : $\frac{L_{\mathrm{h}}}{3}<-L_{\mathrm{O}}<\frac{L_{\mathrm{h}}}{2}$, R4: $\frac{L_{\mathrm{h}}}{2}<-L_{\mathrm{O}}<L_{\mathrm{h}}, \mathrm{R} 5$ : $-L_{\mathrm{O}} \geq L_{\mathrm{h}}$. (a) Sketch of hinge bending, (b) variation of slope along hinge, and (c) deflection along hinge for the different regimes.

with hinge offset of $-0.28 \mu \mathrm{m}$ in Fig. 2(c), right) or always negative (e.g.: deflection curve with hinge offset of $-0.34 \mu \mathrm{m}$ in Fig. 2(c), right). The hinge offset of $-0.42 \mu \mathrm{m}$ (i.e. $L_{\mathrm{o}}=$ $-\frac{L_{\mathrm{h}}}{2}$ ) is the limiting value for this regime, and results in no rotation, as the tip of the hinge ends up perfectly vertical, although the deflection is non-zero. As the final slope is in the direction of rotation, the arc described by the beam is approximated by $S_{1}+S_{2}$ where $S_{1}=L_{\mathrm{a}} \theta_{\mathrm{a}}$ and $S_{2}=0$ for $\theta_{\mathrm{a}}=\frac{F_{\mathrm{x} 1}}{E I}\left(L_{\mathrm{o}} L_{\mathrm{h}}+\frac{L_{\mathrm{h}}^{2}}{2}\right)$.

4) R4-Negative Hinge Offsets: $L_{\mathrm{o}}<0, L_{\mathrm{h}} / 2<\left|L_{\mathrm{o}}\right|<$ $L_{\mathrm{h}}$ : Now, the bigger section of the hinge experiences a negative bending moment. The hinge starts off bending to the left, and at some point along the length of the hinge, experiences a positive bending moment which causes the slope to reduce, but not enough to become positive. The difference with the former regime is that the net slope is now either zero (see Fig. 2(c), $L_{0}=-0.42 \mu \mathrm{m}$ ) or always negative (eg: Fig. 2(c), $L_{\mathrm{o}}=-0.6 \mu \mathrm{m}$ ). This results in anticlockwise rotation, and the arc described by the beam is $S_{1}+S_{2}$ where $S_{1}=L_{\mathrm{a}} \theta_{\mathrm{a}}$ and $S_{2}=0$ for $\theta_{\mathrm{a}}=\frac{F_{\mathrm{x} 1}}{E I}\left(L_{\mathrm{o}} L_{\mathrm{h}}+\frac{L_{\mathrm{h}}^{2}}{2}\right)$.

5) R5-Negative Hinge Offsets: $L_{\mathrm{o}}<0,\left|L_{\mathrm{o}}\right|>L_{\mathrm{h}}$ : Now, all of the hinge experiences a negative bending moment. However, the bending moment becomes progressively less, without ever becoming zero, see Fig. 2(a), R5. This results in anticlockwise rotation again. The hinge offset of $-0.84 \mu \mathrm{m}$ (i.e. $L_{\mathrm{o}}=L_{\mathrm{h}}$ ) is the point at which this regime begins. The expression for the arc $S_{1}+S_{2}$ described by the beam is $S_{1}=$ $L_{\mathrm{a}} \theta_{\mathrm{a}}$ and $S_{2}=0$ for $\theta_{\mathrm{a}}=\frac{F_{\mathrm{x} 1}}{2 E I}\left(L_{\mathrm{o}} L_{\mathrm{h}}+\frac{L_{\mathrm{h}}^{2}}{2}\right)$. The difference in the expression for the arc compared to regime R1 is due 
TABLE II

SUMMARY OF REGIMES OF OPERATION

\begin{tabular}{l|l|l|l|l|l|l} 
Regime & $L_{\mathrm{o}}$ & $\theta_{\mathrm{a}}$ & $\theta_{\mathrm{b}}$ & $S_{1}$ & $S_{2}$ & Direction \\
\hline $\mathrm{R} 1$ & $L_{\mathrm{o}} \geq 0$ & $\frac{F_{\mathrm{x} 1}}{E I}\left(L_{\mathrm{o}} L_{\mathrm{h}}+\frac{L_{\mathrm{h}}^{2}}{2}\right)$ & 0 & $\left(L_{\mathrm{h}}+L_{\mathrm{a}}\right) \frac{\theta_{\mathrm{a}}}{2}$ & $L_{\mathrm{a}} \frac{\theta_{\mathrm{a}}}{2}$ & Clockwise \\
$\mathrm{R} 2$ & $0<-L_{\mathrm{o}}<\frac{L_{\mathrm{h}}}{3}$ & $\frac{F_{\mathrm{x} 1}}{E I}\left(L_{\mathrm{o}} L_{\mathrm{h}}+\frac{L_{\mathrm{h}}^{2}}{2}\right)$ & $\frac{3 F_{\mathrm{x} 1}}{2 E I} L_{\mathrm{o}}^{2}$ & $\left(L_{\mathrm{h}}-3\left|L_{\mathrm{o}}\right|+L_{\mathrm{a}}\right) \frac{\theta_{\mathrm{a}}+\theta_{\mathrm{b}}}{2}$ & $L_{\mathrm{a}} \frac{\theta_{\mathrm{a}}-\theta_{\mathrm{b}}}{2}$ & Clockwise \\
$\mathrm{R} 3$ & $\frac{L_{\mathrm{h}}}{3}<-L_{\mathrm{o}}<\frac{L_{\mathrm{h}}}{2}$ & $\frac{F_{\mathrm{x} 1}}{E I}\left(L_{\mathrm{o}} L_{\mathrm{h}}+\frac{L_{\mathrm{h}}^{2}}{2}\right)$ & $\mathrm{n} / \mathrm{a}$ & $L_{\mathrm{a}} \theta_{\mathrm{a}}$ & 0 & Clockwise \\
$\mathrm{R} 4$ & $\frac{L_{\mathrm{h}}}{2}<-L_{\mathrm{o}}<L_{\mathrm{h}}$ & $\frac{F_{\mathrm{x} 1}}{E I}\left(L_{\mathrm{o}} L_{\mathrm{h}}+\frac{L_{\mathrm{h}}^{2}}{2}\right)$ & $\mathrm{n} / \mathrm{a}$ & $L_{\mathrm{a}} \theta_{\mathrm{a}}$ & 0 & Anticlockwise \\
$\mathrm{R} 5$ & $-L_{\mathrm{o}} \geq L_{\mathrm{h}}$ & $\frac{F_{\mathrm{x} 1}}{E I}\left(L_{\mathrm{o}} L_{\mathrm{h}}+\frac{L_{\mathrm{h}}^{2}}{2}\right)$ & $\mathrm{n} / \mathrm{a}$ & $L_{\mathrm{a}} \theta_{\mathrm{a}}$ & & Anticlockwise \\
\hline
\end{tabular}

(a)

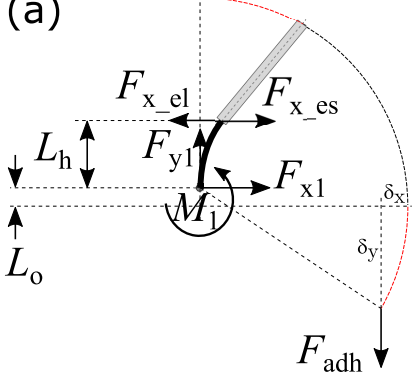

(b)

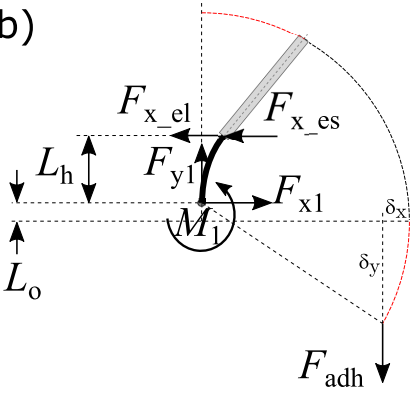

Fig. 3. Forces in reprogramming: (a) Volatile relay that pulls out when actuation is reduced, electrostatic force $F_{\mathrm{es}}$ acts against the elastic force $F_{\mathrm{el}}$. (b) Non-volatile device that stays switched when actuation is completely removed, pull-out requires actuation of the opposite gates; electrostatic force $F_{\text {es }}$ acts with the elastic force $F_{\text {el }}$.

to the fact that the bending moment is uniformly negative, but has the highest magnitude at the anchor point and the least magnitude at the top end of the hinge, as it progressively becomes less negative the further the distance from the anchor. Thus, the slope becomes smaller along the length of the hinge. Whereas in regime R1, the magnitude of the bending moment progressively increases with increasing distance from the anchor point, resulting in an increasing slope.

Finally, for all regimes, the vertical displacement of the tip of the beam can be approximated by the $\operatorname{arc} S_{1}+S_{2}$, as the contact airgap $c$ is much smaller than the radius of the beam semicircle $r_{\mathrm{n}}\left(r_{\mathrm{n}} \approx 50 \times c\right)$, summarized in Table II.

\section{Reprogramming}

The reprogramming voltage depends on both the adhesion force and the elastic force exerted by the hinge as a result of its deformation when the beam tip is in contact with the drain. The stiffness of a rectangular cantilever for a point force applied at the tip is:

$$
k=\frac{3 E I}{L_{\mathrm{h}}{ }^{3}}
$$

Therefore, the equivalent point force at the top of the hinge that results in the same deflection as via gate actuation at voltage $V_{G}$ is given by $F_{\mathrm{X}_{-} \text {es }}=k x$, where the deflection $x$ is given by eq. (7), resulting in

$$
F_{\mathrm{X} \_ \text {es }}=\frac{F_{\mathrm{x} 1 \_V_{G}}\left(3 L_{\mathrm{o}} L_{\mathrm{h}}{ }^{2}+L_{\mathrm{h}}{ }^{3}\right)}{2 L_{\mathrm{h}}{ }^{3}} .
$$

Thus, the system of electrostatic forces applied by gate actuation can be replaced by a single point force $F_{\mathrm{X}_{-} \text {es }}$ applied horizontally at the top of the hinge.

1) Volatile Behavior: The defining characteristic of a volatile relay is that when the actuation voltage is entirely removed, the adhesion force at the tip is not sufficient to keep the relay switched against the opposing elastic force (see Fig. 3(a)). The magnitude of the elastic force $F_{\mathrm{X}_{-} \text {el }}$ exerted after rotation to one of the drains (termed programming), at voltage $V_{\mathrm{pr}}$, is given by eq. (11), where $F_{\mathrm{x} 1_{-} \mathrm{V}_{\mathrm{G}}}=F_{\mathrm{x} 1_{-} \mathrm{V}_{\mathrm{pr}}}$ :

$$
F_{\mathrm{X} \_\mathrm{el}}=\frac{F_{\mathrm{x} 1_{-} \mathrm{V}_{\mathrm{pr}}}\left(3 L_{\mathrm{o}} L_{\mathrm{h}}{ }^{2}+L_{\mathrm{h}}{ }^{3}\right)}{2 L_{\mathrm{h}}{ }^{3}} .
$$

Now, the actuation voltage is progressively reduced, with a corresponding reduction in $F_{\mathrm{X}_{-} \text {es }}$, until the relay pulls out. Taking moments about the hinge anchor in Fig. 3(a),

$$
\begin{aligned}
& M_{1}=F_{\text {adh }} \times\left(r_{\mathrm{o}}-\delta x\right)+F_{\mathrm{X} \_ \text {es }} \times L_{\mathrm{h}}-F_{\mathrm{X} \_\mathrm{el}} \times L_{\mathrm{h}}, \\
& M_{1} \approx F_{\text {adh }} \times r_{\mathrm{o}}+F_{\mathrm{X} \_ \text {es }} \times L_{\mathrm{h}}-F_{\mathrm{X} \_\mathrm{el}} \times L_{\mathrm{h}}
\end{aligned}
$$

as $\delta x \ll r_{0}$. If $M_{1}>0$, the relay pulls out.

2) Non-Volatile Behavior: In non-volatile devices, the adhesion force is large enough to keep the beam tip in contact after the actuation voltage is completely removed. Thus, to cause the relay to pull out, a voltage needs to be applied to the opposing pair of gates to induce an electrostatic force that aids the elastic force in the hinge (see Fig. 3(b)). The gate voltage in this case is referred to as $V_{\text {re }}$ (for reprogramming), and the corresponding electrostatic force is

$$
F_{\mathrm{X} \_ \text {es }}=\frac{F_{\mathrm{x} 1 \_\mathrm{V}_{\mathrm{re}}}\left(3 L_{\mathrm{o}} L_{\mathrm{h}}{ }^{2}+L_{\mathrm{h}}{ }^{3}\right)}{2 L_{2}{ }^{3}} .
$$

The elastic force is unchanged from the volatile case and is still given by the expression in eq. (12). Now the moment balance is given by

$$
M_{1} \approx F_{\text {adh }} \times r_{\mathrm{o}}-F_{\mathrm{X} \_ \text {es }} \times L_{\mathrm{h}}-F_{\mathrm{X} \_ \text {el }} \times L_{\mathrm{h}}
$$

for $\delta x \ll r_{\mathrm{o}}$. Here too, if $M_{1}>0$, the relay pulls out.

\section{Model VAlidation}

We have validated the critical aspects of the analytical model against finite-element simulations carried out in Ansys. The programming voltage was calculated by a MATLAB script 
that incremented the actuation voltage until the arc of rotation $S_{1}+S_{2}$ as defined in Table II exceeded the contact airgap $c$. The model predicted programming voltages are plotted as dots (legend 'Uncal' for 'uncalibrated') and compared with finite-element simulations in Fig. 4(a). As can be seen, the analytical model captures the different regimes of operation and the trends with good accuracy, but produces values that are shifted from the true values. We believe that the error in the model is caused mostly by the approximations in the geometry of rotation and the representation of the actuation forces by unvarying point forces that act through the arcuate centre of the relay as the beam rotates. In actuality, the rotation is slightly elliptical (more pronounced at higher offsets), and the actuation forces are distributed along the length of the actuation airgap.

The accuracy can be improved significantly by introducing a simple empirical correction factor to modulate the arc length $S_{1}+S_{2}$ as $\left(S_{1}+S_{2}\right) \times k_{\mathrm{c}}$ for regimes $\mathrm{R} 1, \mathrm{R} 2$ and $\mathrm{R} 3$ (clockwise rotation), and $\left(S_{1}+S_{2}\right) \times k_{\mathrm{a}}$ for regimes $\mathrm{R} 4$ and $\mathrm{R} 5$ (anticlockwise rotation), where $k_{\mathrm{c}}=w / 80$ and $k_{\mathrm{a}}=w / 120$ (for $w$ in $\mathrm{nm}$ ). The resulting model predictions are plotted as circles with legend 'Cal.' for 'calibrated' in Fig. 4(a), where the absolute fit with the finite-element simulations is much better, while the regimes and trends are still captured accurately. It should be noted that the correction factor, which is dependent on the actuation airgaps and hinge dimensions, is simple enough to ensure it does not detract from the physical insight provided by the model. Finally, at the heart of the model is the deflection along the length of the hinge, which defines the distinct regimes of operation. The deflections along the hinge length for a hinge width of $w=120 \mathrm{~nm}$ at the programming voltage, are shown in Fig. 4(b), for a range of offsets representative of the different regimes. The plots show a good match and verify the change in deflection slope.

\section{EXPERIMENTAL RESULTS}

Test devices were fabricated on a silicon-on-insulator wafer with a $300 \mathrm{~nm}$ thick silicon device layer and $400 \mathrm{~nm}$ thick buried oxide layer using a single e-beam lithography step on a Raith Voyager system, followed by an inductively coupled plasma (ICP) etch of the silicon device layer using an Oxford Instruments Cobra 100 system. The devices were suspended through removal of the buried oxide layer using a vapour phase hydrogen fluoride etch. Finally, a layer of titanium was thermally evaporated as the contact material. The fabricated designs included three different hinge offsets $\left(L_{1}=1.2,0.4\right.$ and $-1 \mu \mathrm{m}$, and two drain electrode contact designs comprising flat (labelled ' $\mathrm{T}$ ') and sprung (labelled ' $\mathrm{S}$ ') electrodes (see Fig. 5(a)) with beam tips that ranged from 50 to $300 \mathrm{~nm}$ wide. The remaining dimensions of the fabricated devices are given in Table I. Here we report the results from experiments using devices fabricated in two separate batches, where the only difference was the duration of the final $\mathrm{Ti}$ deposition step, to yield a thicker contact layer for batch two.

The batch one devices were measured under atmospheric, room temperature conditions with $10 \mathrm{~V}$ applied to the drain,
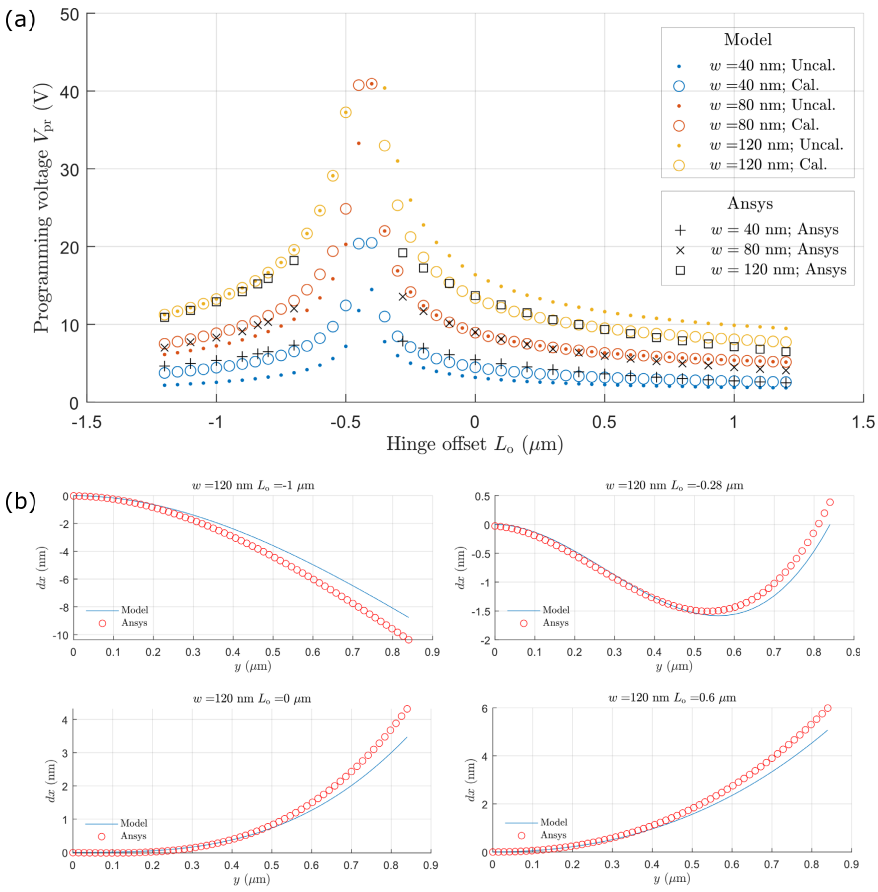

Fig. 4. Comparison of model with finite-element simulations in Ansys. (a) Programming voltage: Uncalibrated and calibrated model values are denoted respectively by dots and circles, and have the same colour for a given hinge width. (b) Hinge deflection for hinge width $w=120 \mathrm{~nm}$ for a range of hinge offsets representative of the different regimes.

while the batch two devices were measured in vacuum at $200{ }^{\circ} \mathrm{C}$ with a drain voltage of $2 \mathrm{~V}$. These voltages were chosen to give a drain current at least two orders of magnitude greater than the noise floor, which was a few $\mathrm{pA}$ for a static voltage (applied to the drain) on the Agilent B1500 semiconductor parameter analyser used for measurements. The thicker contact coating for the second batch resulted in a lower contact resistance, and thus we could apply a lower drain voltage. The measured programming voltages for fabricated devices with designed hinge offsets of $-1,0.4$ and $1.2 \mu \mathrm{m}$, and a hinge width of $100 \mathrm{~nm}$ are shown in Fig. 5(b), along with the analytical model predictions. We observed variation of the nominal hinge width across the die, and thus extracted the fabricated hinge width from SEM images, with an estimated error of $\pm 5 \mathrm{~nm}$ (due to uncertainty in extracting the length from the image) which determine the error bars for the model. As can be seen, the measurements match up well with the model predictions, including the anticlockwise rotation for the negative offset of $-1 \mu \mathrm{m}$.

We also successfully reprogrammed several non-volatile devices. ${ }^{1}$ First, the reprogramming voltages over several consecutive cycles for a device from Batch 2 with a hinge offset $L_{\mathrm{o}}=1.2 \mu \mathrm{m}$, hinge width $w \approx 80 \mathrm{~nm}$, beam tip width $c_{\mathrm{w}}=50 \mathrm{~nm}$ and drain type "T" are shown in Fig. 5(c). Second, the surface adhesion forces estimated from mea-

\footnotetext{
${ }^{1}$ We continuously cycled seven devices in the high-temperature measurements up to a maximum of 42 times, and four devices in the room temperature measurements for a maximum of 20 times before the contact degraded and the current dropped. We also carried out data retention tests on two devices after storing the devices in an ordinary gel pack for six months; these devices retained the programmed state, and could be reprogrammed.
} 
(a)

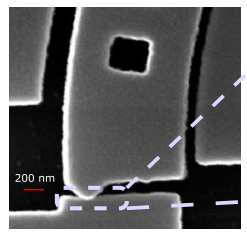

T-type drain (50T)
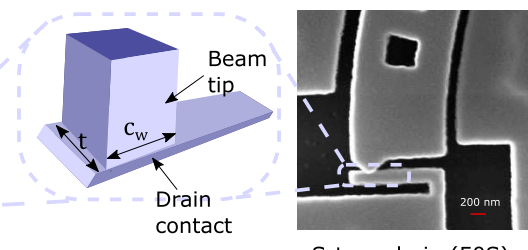

S-type drain (50S)

(b)

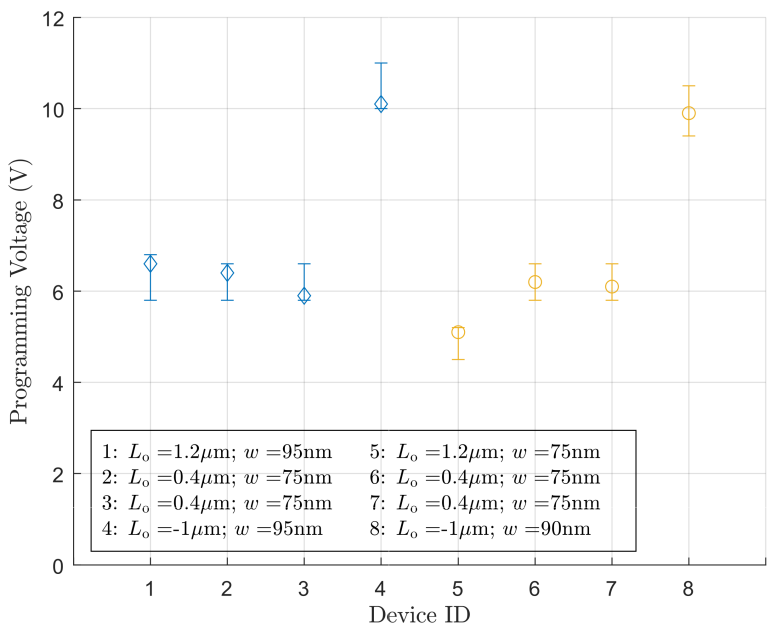

(c)

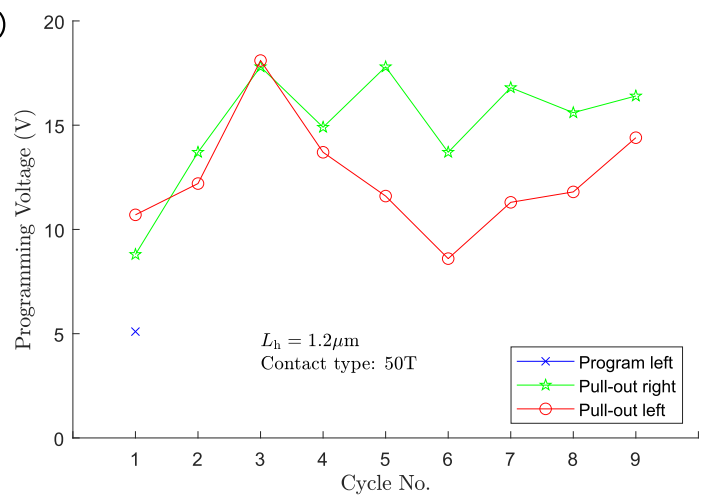

(d)

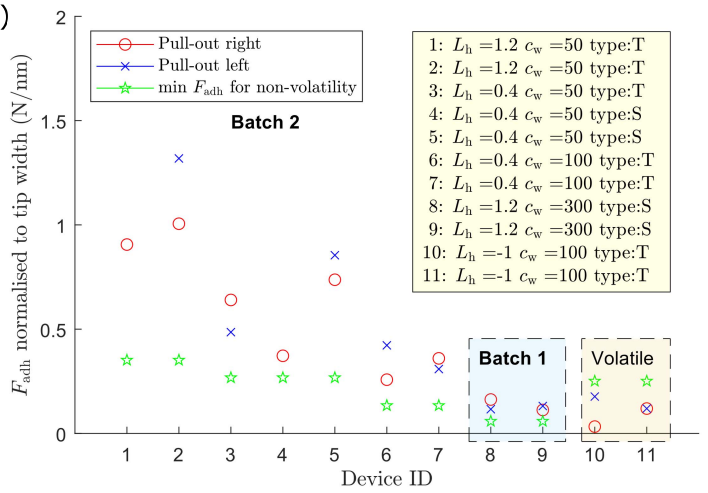

Fig. 5. Experimental results. (a) Different contact designs. (b) Comparison of model with measurements of programming voltage (c) Evolution of pull-out voltage for a single relay (Device ID 2) with cycling. (d) Adhesion force computed from experimentally measured pull-out voltages for different relays from batches one and two.

sured pull-out voltages for the first reprogramming cycle for 11 separate devices from both batches are shown in Fig. 5(d) (the first cycle is chosen as the Ti-coated contact electrodes tend to degrade quickly with cycling). The adhesion forces are calculated from the system of equations defined by (13) and (15) for volatile and non-volatile behaviour respectively, by setting the net moment $M_{1}$ to zero, and using eq. (11) at the pull-out voltage to calculate the electrostatic force $F_{\mathrm{X} \_ \text {es }}$ and eq. (12) to calculate the elastic force $F_{\mathrm{X} \_ \text {el }}$. Surface adhesion at the contacts can be attributed to van der Waals forces, electrostatic forces due to the applied bias, and capillary forces from contaminants [15]-[17]. As the applied drain bias is different between the two, the electrostatic component of the surface adhesion force is different, while an ambient temperature of $200{ }^{\circ} \mathrm{C}$ is likely to cause any moisture to evaporate and reduce the effect of capillary forces. Thus, only the measurements within a batch can be compared. Assuming capillary effects are broadly similar across different experiments within a given testing environment, normalising the adhesion force to contact surface area allows us to compare between different contact designs, as to a first order, the van der Waals and electrostatic forces are proportional to the contact area. The surface adhesion forces are calculated by using the experimentally measured pull-out voltages, and the calculated elastic force in the hinge and beam under the strain corresponding to the tip being in contact with the drain, i.e. the system of equations (11), (12), (13), (14), (15). Also shown in the figure are the model predicted minimum adhesion forces required to ensure non-volatility for each device. The forces are normalised by dividing the force by the width of the beam tip, giving units of $\mathrm{N} \mathrm{nm}^{-1}$. As the beam tip thickness $t$ is constant for all devices (defined by the thickness of the SOI wafer device layer), this force is interpreted as the adhesion force per unit length $c_{\mathrm{W}}$ for a contact surface area $t \times c_{\mathrm{W}}$.

The model correctly predicts whether every device in Fig. 5(d) behaves as a volatile or non-volatile device. Devices 1 through 9 exhibited non-volatile behaviour in measurements and the difference between the measured value and theoretically calculated minimum value represents the available margin before the device becomes volatile. Devices 10 and 11 have a negative hinge offset $\left(L_{\mathrm{o}}=-1 \mu \mathrm{m}\right)$, and exhibited volatile behaviour in measurements, this time with the theoretical minimum adhesion force for non-volatile behaviour being greater than the measured value.

\section{Discussion}

The measured pull-out voltages show cycle-to-cycle variation for a given device (Fig. 5(c)), implying a varying adhesion force at the contact, as the electromechanical properties of the hinge are unlikely to change across a few cycles. We believe the change in adhesion force is primarily caused by the effective contact area changing from cycle to cycle, due to the surface roughness and lack of uniformity in the deposited contact layer (the presence of surface contaminants could also have been a contributing factor). Similarly, while a constant adhesion force per unit area can be expected between devices with similar contact designs under ideal conditions, the measurements indicate the effective contact area varies 


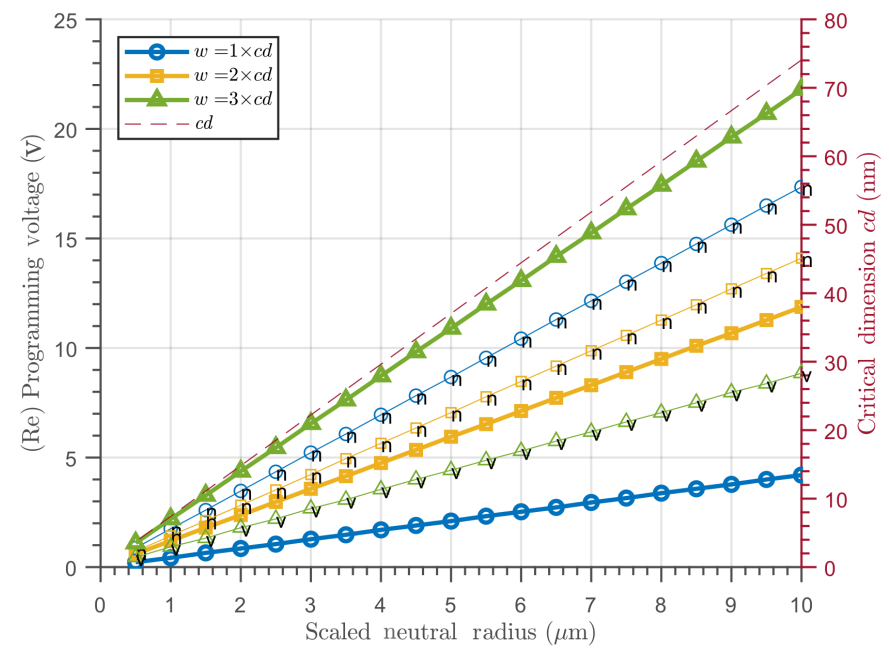

Fig. 6. Scaling study where all dimensions are scaled proportionately. The critical dimension associated with a given arc radius is shown on the right, with programming and reprogramming voltages on the left. ' $\mathrm{n}$ ' and ' $\mathrm{v}$ ' denote non-volatile and volatile operation.

(Fig. 5(d)), due to the same effect. It can be seen, though, that the type ' $\mathrm{S}$ ' contacts consistently exhibit a lower surface adhesion force for the same area. Generally, we expect the nature of the contact made by the sprung (type ' $S$ ') and solid (type ' $\mathrm{T}$ ') contacts to be different as the sprung contacts undergo strain on making contact. Thus, part of the applied electrostatic energy is stored as strain energy in the contact spring, effectively reducing the force between the contacting surfaces, which would result in a lower effective contact area and a correspondingly lower adhesion force. This hypothesis is consistently borne out by the results shown in Fig. 5(d).

The main functional parameters for our relay are whether it is volatile or non-volatile and the programming and pullout voltages, $V_{\mathrm{pr}}$ and $V_{\mathrm{po}}$. The main design parameters are the beam inner and outer radii $r_{\mathrm{o}}$ and $r_{\mathrm{i}}$, hinge width $w$, hinge offset $L_{1}$, actuation (gate) gap $g$, contact gap $c$, contact type (' $\mathrm{T}$ ' or ' $\mathrm{S}$ '), and contact width $c_{\mathrm{W}}$ (it is also possible to have a different hinge type such as a softer, serpentine hinge, but this simply changes the hinge stiffness). Our model shows that, for the fabricated devices, the minimum adhesion force per contact width to achieve non-volatility is of the order of $0.2 \mathrm{~N} / \mathrm{nm}$ to $0.4 \mathrm{~N} / \mathrm{nm}$. Using an adhesion force of $0.4 \mathrm{~N} / \mathrm{nm}$, we have carried out a scaling study to quantify the effect of reducing the arc radii while scaling the other dimensions proportionately.

The results of this study are plotted in Fig. 6. The x-axis shows the scale of the device while the critical dimension (hinge width) associated with it is shown on the right. Under a fixed device silicon layer thickness and proportionate scaling (i.e. maintaining the same ratio of dimensions as our fabricated devices), the programming voltage shows an approximately linear dependence on the arc radius. As the second moment of area $I$ is inversely proportional to $w^{3}$, scaling causes it to reduce with a cubic dependence. The rotation angle $\theta$ in eq. (9) also depends on the force $F_{\mathrm{x} 1}$, which decreases linearly with scaling according to eq. (1). As the force is proportional to the square of the voltage, the net result is an approximate linear reduction in the programming voltage with scaling.

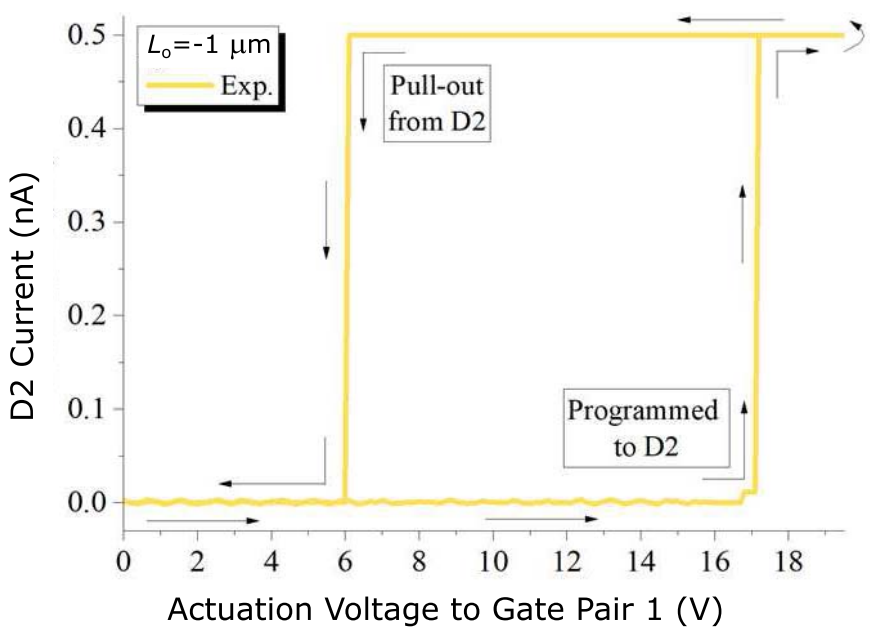

Fig. 7. Hysteresis plot of device with hinge offset $L_{\mathrm{O}}=-1 \mathrm{~mm}$, showing no discernible leakage current in the off state.

The reprogramming voltage is dependent on the balance between the restoring hinge elastic force and contact adhesion force, and changing the size of the contact area provides a means to change between volatile and non-volatile functionality, and tailor the reprogramming voltage to be equal to or less than the programming voltage, a valuable and unique attribute in non-volatile memory.

\section{SUMMARY AND CONCLUSION}

We have designed, fabricated and characterised a range of relays with a rotational architecture in order to better understand its stiction-based non-volatile behaviour and developed an accurate, physics-based model from first principles to analyse its operation and how it scales. The scaling study shows that programming and reprogramming voltages of $\approx 1 \mathrm{~V}$ can be achieved with a critical dimension of $\approx 10 \mathrm{~nm}$ and a device footprint under $\approx 2 \mu \mathrm{m}^{2}$. When combined with a stable contact material such as nanocrystalline graphite [5], the models and methods presented in this work enable development of high-temperature, radiation-hard non-volatile memory with zero standby-power, demanded by emerging applications such as autonomous sensor nodes in the IoT.

\section{APPENDIX A}

\section{Hysteresis Plot AND LEAKAge CuRRent}

In order to test the leakage current, we set a (low) current compliance of $500 \mathrm{pA}$ in the semiconductor parameter analyser (Agilent B1500) in our measurement setup and tested a device that had an offset of $L_{0}=-1 \mathrm{~mm}$ at $200{ }^{\circ} \mathrm{C}$. This relay exhibited volatile behaviour, rotating to drain D2 at $16.8 \mathrm{~V}$ when actuated by gate pair PG2 / AG2, and pulling out when the actuation is reduced to $6.2 \mathrm{~V}$ (see Fig. 8).

\section{APPENDIX B}

\section{ROTATIONAL SENSITIVITY OF RELAY}

We have extracted the rotational sensitivity of the device from finite-element simulations using representative values of $w=80,120 \mathrm{~nm}$ and hinge offset $L_{\mathrm{o}}=0.2 \mu \mathrm{m}$. The sensitivity 


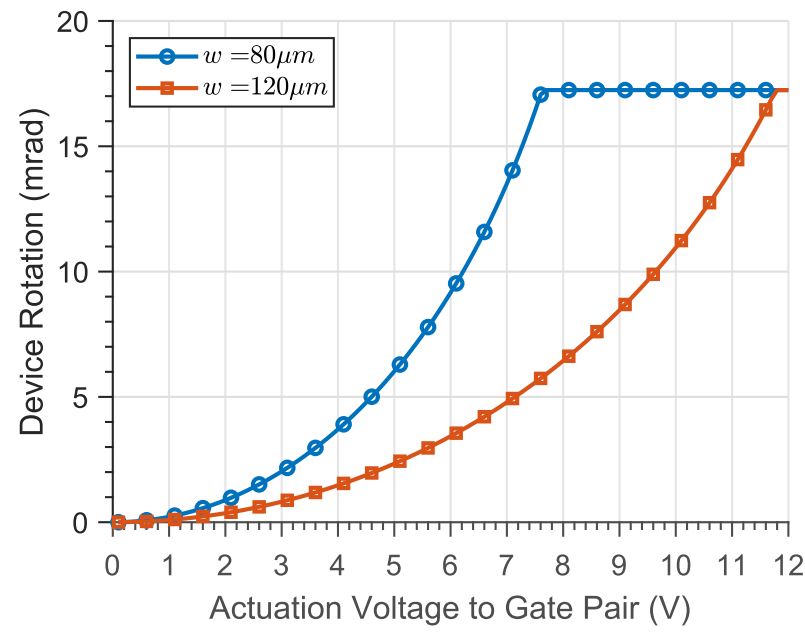

Fig. 8. Rotational sensitivity of the relay for $w=80,120 \mathrm{~nm}$ and offset $L_{\mathrm{o}}=0.2 \mu \mathrm{m}$.

changes as a function of the device as the trajectory of the beam is not perfectly circular (given that the hinge is only an approximation of a perfect pivot). This deviation of the trajectory from a perfect circle, though, is small enough that there is no snap in of the beam as discussed in our previous work [14].

\section{REFERENCES}

[1] O. Y. Loh and H. D. Espinosa, "Nanoelectromechanical contact switches," Nature Nanotechnol., vol. 7, no. 5, p. 283, 2012.

[2] V. Pott, H. Kam, R. Nathanael, J. Jeon, E. Alon, and T.-J. K. Liu, "Mechanical computing redux: Relays for integrated circuit applications," Proc. IEEE, vol. 98, no. 12, pp. 2076-2094, Dec. 2010.

[3] S. Rana et al., "Energy and latency optimization in NEM relay-based digital circuits," IEEE Trans. Circuits Syst. I, Reg. Papers, vol. 61, no. 8, pp. 2348-2359, Aug. 2014.
[4] T. Qin, S. J. Bleiker, S. Rana, F. Niklaus, and D. Pamunuwa, "Performance analysis of nanoelectromechanical relay-based fieldprogrammable gate arrays," IEEE Access, vol. 6, pp. 15997-16009, 2018.

[5] S. Rana et al., "Nano-crystalline graphite for reliability improvement in MEM relay contacts," Carbon, vol. 133, pp. 193-199, Jul. 2018.

[6] A. Bazigos et al., "Analytical compact model in Verilog-A for electrostatically actuated ohmic switches," IEEE Trans. Electron Devices, vol. 61, no. 6, pp. 2186-2194, Jun. 2014.

[7] T.-H. Lee, S. Bhunia, and M. Mehregany, "Electromechanical computing at $500{ }^{\circ} \mathrm{C}$ with silicon carbide," Science, vol. 329, no. 5997, pp. 1316-1318, Sep. 2010.

[8] H. R. Shea, "Effects of radiation on MEMS," Proc. SPIE, vol. 7928 , Feb. 2011, Art. no. 79280E.

[9] P. Singh, D. S. Arya, and U. Jain, "MEM-FLASH non-volatile memory device for high-temperature multibit data storage," Appl. Phys. Lett., vol. 115, no. 4, Jul. 2019, Art. no. 043501.

[10] S. W. Lee, S. J. Park, E. E. B. Campbell, and Y. W. Park, "A fast and low-power microelectromechanical system-based non-volatile memory device," Nature Commun., vol. 2, no. 1, pp. 1-6, Sep. 2011.

[11] P. Singh, G. L. Chua, Y. S. Liang, K. G. Jayaraman, A. T. Do, and T. T.-H. Kim, "Anchor-free NEMS non-volatile memory cell for harsh environment data storage," J. Micromech. Microeng., vol. 24, no. 11, Nov. 2014, Art. no. 115007.

[12] S.-B. Kim, H.-W. Min, Y.-B. Lee, S.-H. Kim, P.-K. Choi, and J.-B. Yoon, "Utilizing mechanical adhesion force as a high contact force in a MEMS relay," Sens. Actuators A, Phys., vol. 331, Nov. 2021, Art. no. 112894

[13] D. S. Arya, S. Kumar, and P. Singh, "A cantilever type bistable MEM nonvolatile memory with piezoelectric deactuation for high-temperature applications," in Proc. IEEE SENSORS, Oct. 2019, pp. 1-4.

[14] S. Rana et al., "Nanoelectromechanical relay without pull-in instability for high-temperature non-volatile memory," Nature Commun., vol. 11, no. 1, pp. 1-10, Dec. 2020.

[15] J. Yaung, L. Hutin, J. Jeon, and T.-J. K. Liu, "Adhesive force characterization for MEM logic relays with sub-micron contacting regions," J. Microelectromech. Syst., vol. 23, no. 1, pp. 198-203, Feb. 2014.

[16] J. Mouro, S. Rana, J. D. Reynolds, H. M. H. Chong, and D. Pamunuwa, "Estimating the surface adhesion force using pull-in/-out hysteresis in comb-drive devices," in Proc. Int. Conf. Solid-State Sens., Actuators Microsystems (TRANSDUCERS), Jun. 2019, pp. 1981-1984.

[17] S. Kumar, D. S. Arya, and P. Singh, "Volatile or non-volatile switching? Establishing design parameters for metal-contact relays using ON/OFF hysteretic behavior (RT to $300{ }^{\circ} \mathrm{C}$ )," Appl. Phys. Lett., vol. 118, no. 1, Jan. 2021, Art. no. 013505. 